\title{
Prediction of Low-Temperature Rheological Properties of SBS Modified Asphalt
}

\author{
Qian Chen, ${ }^{1}$ Chaohui Wang $\mathbb{D}^{1},{ }^{1}$ and Liang Song $\mathbb{D i D}^{2}$ \\ ${ }^{1}$ School of Highway, Chang'an University, Xi'an 710064, China \\ ${ }^{2}$ Xinjiang Transportation Planning Surveying and Design Institute, Urumqi 830006, China \\ Correspondence should be addressed to Chaohui Wang; wchh0205@chd.edu.cn and Liang Song; 3359559@qq.com
}

Received 30 September 2020; Revised 9 November 2020; Accepted 20 November 2020; Published 2 December 2020

Academic Editor: B. Binici

Copyright (c) 2020 Qian Chen et al. This is an open access article distributed under the Creative Commons Attribution License, which permits unrestricted use, distribution, and reproduction in any medium, provided the original work is properly cited.

The extreme learning machine (ELM) algorithm optimized by genetic algorithm (GA) was used to quickly predict the lowtemperature rheological properties of styrenic block copolymer (SBS) modified asphalt through the properties of the raw materials. In this work, one hundred groups of survey data and test data were collected and analyzed. Fourteen vital raw material parameters, such as chemical composition indexes of matrix asphalt and technical indexes of SBS modifier, were selected as the input parameter. The stiffness modulus and $m$-value of SBS modified asphalt were taken as the output parameter. Then, the GAELM prediction model of low-temperature rheological properties was established. According to comparison and analysis with other prediction models, the accuracy and output stability of the GA-ELM prediction model were verified. The results show that the GA-ELM model had obvious accuracy and efficiency. It can be used to predict the low-temperature rheological properties of SBS modified asphalt. Compared with the traditional prediction models, the error of the GA-ELM model was reduced by $68.97-81.48 \%$.

\section{Introduction}

At present, the main research methods of low-temperature performance of styrenic block copolymer (SBS) modified asphalt are the force ductility method, microscopic observation method, and rheological test method [1]. Strategic Highway Research Program (SHRP) mainly uses the bending beam rheological test (BBR) to evaluate the lowtemperature performance of asphalt [2]. The stiffness modulus and creep rate ( $m$-value) of asphalt are the core indexes. Some scholars have carried out a large number of macroexperiments and microanalysis. Shan et al. evaluated the effect of SBS on the linear and nonlinear rheological behavior of asphalt binder [3]. Chen et al. analyzed the characteristics and reasons for the rheological behavior of SBS modified asphalt with different SBS dosages [4]. Ren et al. studied the effect of trans-polyactenamer on rheological properties, microstructure, and thermal stability of crumb rubber (CR)/SBS modified asphalt [5]. The effects of stiffness modulus and $m$-value on low-temperature performance of SBS modified asphalt were determined $[6,7]$. However, due to the complex source of matrix asphalt and SBS modifier and the small number of test samples, there are some differences in the research conclusions of different scholars. For SBS modified asphalt, the chemical composition of matrix asphalt and the physicochemical index of modifier have different effects on the performance of modified asphalt. As a result, many factors need to be considered in the performance evaluation of SBS modified asphalt [8], and the amount and time of the experiment are large relatively, limiting the promotion and application of SBS modified asphalt in the engineering field to a certain extent.

In order to reduce the amount of test and get more accurate results quickly, the energy coefficient method (ECM), grey target decision-making method (GTDM), backpropagation algorithm (BP), radial basis function algorithm (RBF), and other evaluation methods and algorithms were used to predict the performance of asphalt materials [9-13]. Yan et al. predicted the change of modulus 
and phase angle of SBS polymer modified asphalt by exponential regression and linear regression [14]. Xu et al. established the performance prediction model of SBS modified asphalt by using principal component analysis (PCA) and partial least squares (PLS) [15]. Diab et al. predicted the viscosity and rheological behavior of SBS modified asphalt, according to Vinogradov Malkin and Phillips Deutsch models [16]. However, there are some problems with these methods $[17,18]$. For example, it is easy to fall into the local optimum solution in the process of solving; the parameters are not easy to determine; the requirements of training samples are high relatively; and the training is difficult.

Therefore, many vital parameters, such as the chemical composition of matrix asphalt and physicochemical index of modifier, were selected. The extreme learning machine (ELM) algorithm was optimized by the genetic algorithm (GA) to form the GA-ELM algorithm. The prediction model for the low-temperature rheological properties of SBS modified asphalt based on the GA-ELM algorithm was established. Compared with the BP model and the ELM model, the accuracy and output stability of the GA-ELM prediction model were verified. It provided a new pathway for the study of low-temperature rheological properties of SBS modified asphalt.

\section{Experiment}

2.1. Test Method. According to Test Method for Separation of Asphalt into Four Fractions (NB/SH/T 0509-2010), CN, the component of asphalt, was measured [19]. The specific steps were as follows. The asphaltenes were precipitated from the sample with normal heptane. After filtration, the soluble inclusions in the precipitation were removed by reflux of normal heptane. The asphaltenes were obtained by dissolving precipitation with toluene reflux. Then, the deasphalting part was adsorbed on the alumina chromatographic column. In turn, normal heptane, toluene, and tolueneethanol were used to obtain saturates, aromatics, and colloids.

2.2. Data Preparation. The raw material parameters of SBS modified asphalt were selected as the input parameter. They mainly consisted of the following: PG-grade, stiffness modulus, $m$-value, asphaltenes, colloids, aromatics, saturates of matrix asphalt and structure, block ratio $(\mathrm{S} / \mathrm{B})$, tensile strength, elongation, permanent deformation, hardness (HSD), and the content of SBS modifier. The stiffness modulus and $m$-value of SBS modified asphalt were taken as the output parameter.

In order to collect more sample data, on the basis of the data provided in the literature [8], more series of experiments were completed. The test parameters and indexes are shown in Tables 1 and 2. Finally, 100 sets of test data were collected. Among them, 80 sets of data were used for training, and 20 sets were used for testing. According to equation (1), the sample data were normalized.

$$
X_{i}=\frac{X-X_{\min }}{X_{\max }-X_{\min }}
$$

where $X_{i}$ are the normalized sample data, $X$ are the initial sample data, $X_{\max }$ is the maximum of the initial sample data, and $X_{\min }$ is the minimum of initial sample data.

2.3. GA-ELM Prediction Model. ELM is a new feed-forward neural network. Compared with the traditional neural network with a single hidden layer, its hidden layer does not need iteration and has an obvious faster learning speed. However, the input layer weight matrix and the hidden layer threshold matrix of the ELM model are random. GA has a strong global optimization ability. The fitting accuracy of the ELM model can be improved by optimizing the above matrix with the GA. The GA-ELM model was established in the following steps [20].

(1) For input sample $X_{i}$, which had been normalized, the output matrix $(H)$ of hidden layer neurons was calculated according to the following equation:

$$
H=g\left(W X^{T}+b\right)
$$

where $W$ is the weight matrix of the input layer, $b$ is the threshold matrix of the implicit layer, and $g$ is the neuronal activation function of the hidden layer, which was a "sigmoid" function in this study.

(2) According to equation (3), the output value $(P)$ of the ELM neural network was calculated.

$$
P=\left(H^{T} \beta\right),
$$

where $\beta$ is the weight matrix from the implicit layer to the output layer, and the ELM neural network can be determined by calculating $\beta$.

(3) The given training output sample $(Y)$ was used to replace the output value of the neural network. $\beta$ can be obtained by solving the least square solution of the following equation:

$$
\min _{\beta}\left\|H^{T} \beta-Y\right\|
$$

(4) The genetic algorithm was used to find the optimal initial $W$ and $b$ of the ELM neural network. Through fitness function, the genetic algorithm found the corresponding individuals of minimum fitness value through selection, crossover, and mutation operation.

(5) The optimal initial weight and threshold assignment of the ELM neural network were obtained by the genetic algorithm. The number of the hidden layers 
TABle 1: Test parameters and their indexes of matrix asphalt.

\begin{tabular}{|c|c|c|c|c|c|c|c|}
\hline \multirow[b]{2}{*}{ Type } & \multicolumn{7}{|c|}{ Index } \\
\hline & $\begin{array}{l}\text { PG- } \\
\text { grade }\end{array}$ & $\begin{array}{l}\text { Stiffness modulus }\left(-18^{\circ} \mathrm{C}\right) \\
(\mathrm{MPa})\end{array}$ & $\begin{array}{c}m \text {-value }\left(-18^{\circ} \mathrm{C}\right) \\
\left(\mathrm{MPa} \cdot \mathrm{s}^{-1}\right)\end{array}$ & $\begin{array}{c}\text { Asphaltenes } \\
(\%)\end{array}$ & Colloids (\%) & $\begin{array}{l}\text { Aromatics } \\
(\%)\end{array}$ & $\begin{array}{c}\text { Saturates } \\
(\%)\end{array}$ \\
\hline SK 90 & $58-22$ & $270-275$ & $0.295-0.300$ & $8.73-8.78$ & $19.35-19.40$ & $46.65-46.70$ & $25.20-25.24$ \\
\hline Shell 90 & $58-22$ & $325-320$ & $0.270-0.274$ & $8.10-8.14$ & $33.17-33.21$ & $35.55-35.59$ & $23.08-23.13$ \\
\hline Kunlun 90 & $58-22$ & $270-275$ & $0.292-0.295$ & $10.54-10.57$ & $30.14-30.17$ & $39.80-39.83$ & $19.19-19.22$ \\
\hline $\begin{array}{l}\text { Zhenhai } \\
90\end{array}$ & $58-22$ & $265-269$ & $0.288-0.293$ & $7.24-7.28$ & $30.08-30.12$ & $39.62-39.65$ & $23.00-23.03$ \\
\hline Esso 70 & $58-16$ & $311-315$ & $0.190-0.195$ & $15.50-15.52$ & $20.14-20.17$ & $44.20-44.26$ & $20.09-20.13$ \\
\hline
\end{tabular}

TABLE 2: Test parameters and their indexes of SBS modifier.

\begin{tabular}{|c|c|c|c|c|c|c|c|}
\hline \multirow{2}{*}{ Type } & \multicolumn{7}{|c|}{ Index } \\
\hline & Structure & Block ratio $(\mathrm{S} / \mathrm{B})$ & Tensile strength $(\mathrm{MPa})$ & Elongation (\%) & Permanent deformation (\%) & Hardness & Content (\%) \\
\hline DG2 & Star & $40 / 60$ & $>12.0$ & $>650$ & $<30$ & $>79$ & $3.6-5.2$ \\
\hline T161B & Star & $30 / 70$ & $>18.0$ & $>630$ & $<25$ & $>78$ & $3.6-5.2$ \\
\hline $1320-115$ & Linear & $30 / 70$ & $>18.0$ & $>750$ & $<40$ & $>70.5$ & $3.6-5.2$ \\
\hline YS4303 & Star & $30 / 70$ & $>12.0$ & $>590$ & $<45$ & $>65$ & $3.6-5.2$ \\
\hline YH791 & Linear & $30 / 70$ & $>18.0$ & $>700$ & $<45$ & $>60$ & $3.6-5.2$ \\
\hline
\end{tabular}

Note. $\mathrm{S} / \mathrm{B}$ is the abbreviation of styrene and butadiene. Test temperature was $-18^{\circ} \mathrm{C}$.

was determined, and the GA-ELM model was established.

(6) According to equations (5)-(7), mean absolute error (MAE), mean absolute percent error (MAPE), and root mean squared error (RMSE) were used as error criteria. The GA-ELM model was tested and evaluated by the test set samples. The algorithm flow is shown in Figure 1 [20].

$$
\begin{aligned}
\text { MAE } & =\frac{1}{n} \sum_{i=1}^{n}\left|P-P^{\prime}\right|, \\
\text { MAPE } & =\frac{1}{n} \sum_{i=1}^{n}\left|\frac{P-P^{\prime}}{P} \times 100 \%\right|, \\
\text { RMSE } & =\sqrt{\frac{1}{n} \sum_{i=1}^{n}\left(P-P^{\prime}\right)^{2},}
\end{aligned}
$$

where $P$ is the true value, $P^{\prime}$ is a predictive value, and $n$ is the number of test sample data.

\section{Results and Discussion}

3.1. Parameter Optimization of the ELM Model. The key operation parameter of the ELM model is the number of hidden layers. The operation parameters of the genetic algorithm include population size, crossover probability, mutation probability, and maximum iterations. In practical applications, it is often necessary to undergo a large number of tests before the reasonable range of these parameters is determined. Based on the test set data, the superior hidden layer number of the ELM prediction model was determined through multiple tests, as shown in Figure 2.

Figures 2(a) and 2(b) show that with the increase of the hidden layer number in the ELM model, the error decreased first and then increased. When the number of the hidden layers was in the range of 60-70, the error was small. After many tests, the optimal hidden layer number of the ELM model was determined to be 65 . At this time, MAE, MAPE, and RMSE were $369.37 \%, 1.66 \%$, and $502.77 \%$, respectively. It was noteworthy that MAE and RMSE were large and needed to be reduced in the subsequent optimization process. For $m$-value, Figures $2(\mathrm{c})$ and 2(d) show that the optimal hidden layer number was determined to be 55 . Its MAE, MAPE, and RMSE were $0.27 \%, 0.85 \%$, and $0.32 \%$, respectively.

3.2. Parameter Optimization of the GA. After repeated cyclic tests, the optimal values of the four parameters of the GA were determined by using the Sheffield toolbox in MATLAB software, as shown in Figure 3. To simplify the simulation process, when population size, crossover probability, and mutation probability were optimized, the maximum iterations was set to 50 .

Figure 3 indicates that with the change of the four parameters of the GA, the corresponding error values also changed regularly. In general, the recommended ranges of population size, crossover probability, mutation probability, and maximum iterations are 40-100, 0.40-0.80, 0.001-0.1, and 100-300, respectively. According to the test results of the stiffness modulus prediction model, the optimal ranges of population size, crossover probability, mutation probability, and maximum iterations were 40-45, 0.40-0.50, 0.0005-0.001, and 300-400, respectively. As for the $m$-value prediction model, the optimal ranges of population size, 


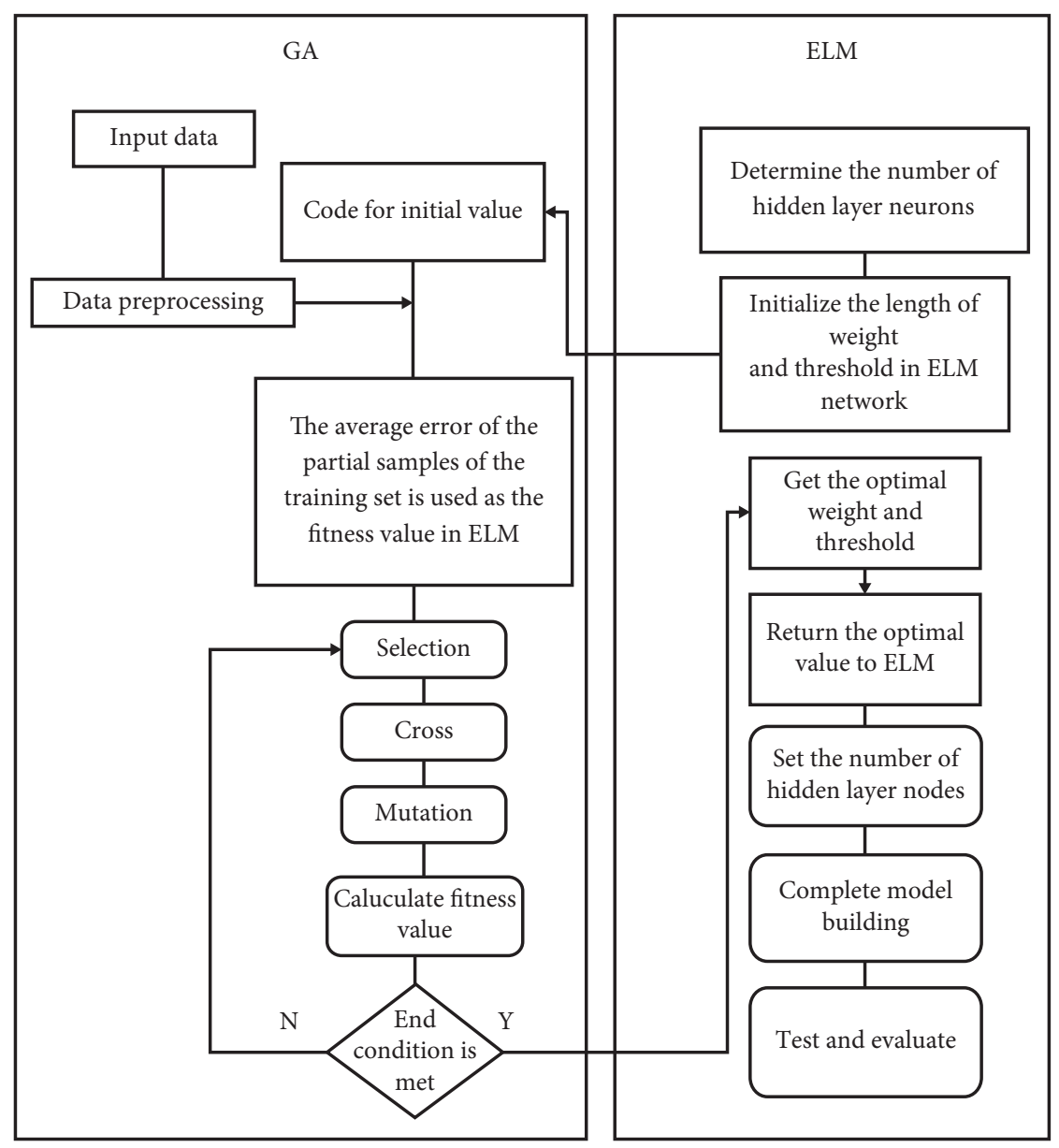

Figure 1: The algorithm flow of the GA-ELM model.

crossover probability, mutation probability, and maximum iterations were $45-50,0.70-0.80,0.0005-0.001$, and 200-300, respectively. At this time, the genetic algorithm has better convergence to the optimal weights and thresholds. Due to the large scale of the weight matrix and threshold matrix, their specific values are no longer described.

3.3. Prediction Accuracy of the GA-ELM Model. The Pearson correlation test was used to verify the prediction accuracy of the GA-ELM model. The discriminant coefficient $\left(R^{2}\right)$ of the fitting function between the true value and predictive value of the test set sample was calculated, as shown in Figure 4. The accuracy of the GA-ELM prediction model for lowtemperature rheological properties was determined by analyzing the goodness of fit between predictive data and true data.

Figure 4 shows that the true value of test set samples is very close to the predictive value. The correlation coefficients $(R)$ of the fitting function of the two models are 0.9997 and 0.9992 , respectively. This indicated that the predictive value was strongly correlated with the true value, and the prediction accuracy of the prediction model was high. In addition, the corresponding discriminant coefficients $\left(R^{2}\right)$ are 0.9995 and 0.9985 , respectively, which indicates that the GA-
ELM prediction model could maintain the stability of output based on high prediction accuracy.

3.4. Contrastive Analysis of Different Models. To further verify the output stability of the GA-ELM model, the BP model, the ELM model, and the GA-ELM model were used to predict 20 sets of test sample data. For stiffness modulus, after many simulation calculations, the neuron node number of hidden layers in the BP model was set to 15 . The hidden layer number in the ELM model was set to 65. The population size, crossover probability, mutation probability, and maximum iterations of the GA were set to 45 , $0.80,0.001$, and 350 , respectively. And for the $m$-value, the neuron node number of hidden layers in the BP model was set to 15. The hidden layer number in the ELM model was set to 55. The population size, crossover probability, mutation probability, and maximum iterations of the GA were set to $50,0.50,0.001$, and 200 , respectively. The prediction results based on different models are shown in Figure 5.

Figure 5 shows that the three models achieved consistent prediction results. Still, the prediction error of the GA-ELM model was significantly smaller than that of the BP model and the ELM model. For stiffness modulus, the MAE, MAPE, and RMSE of the GA-ELM model were 


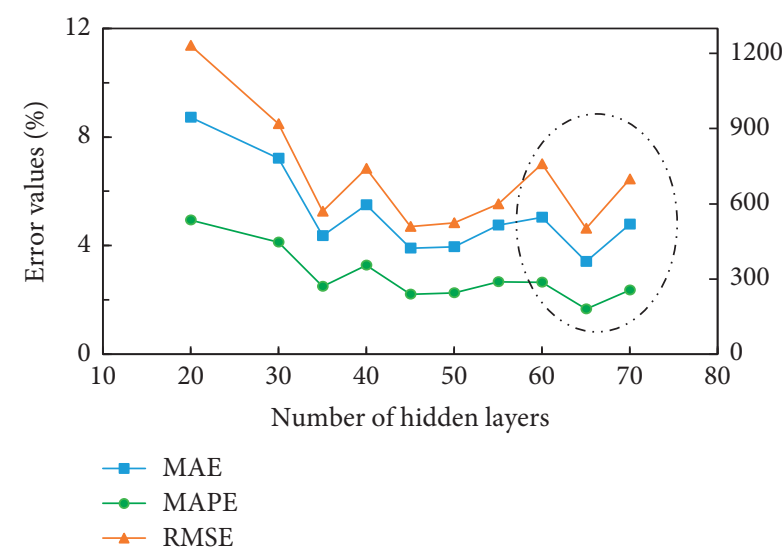

(a)

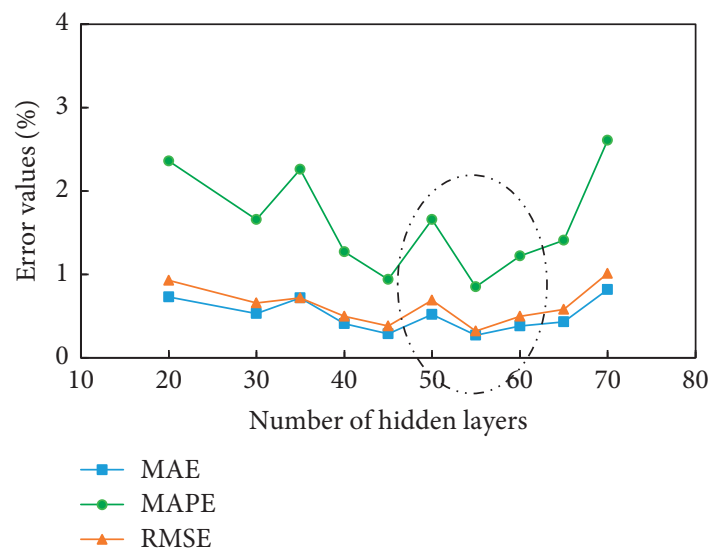

(c)

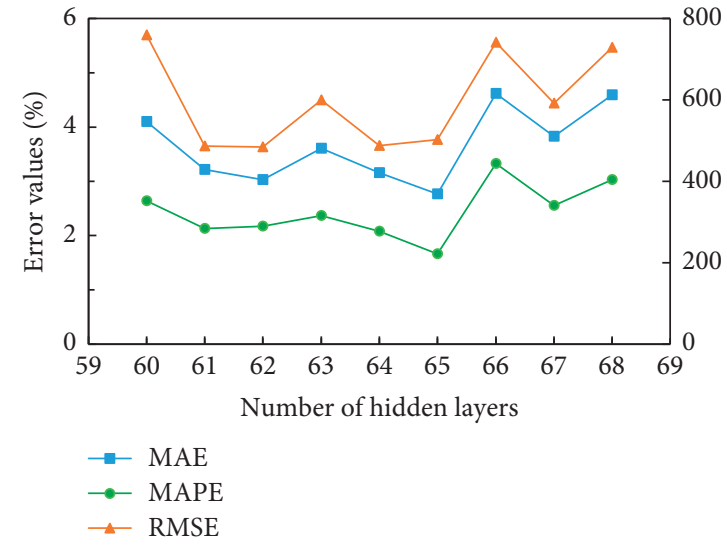

(b)

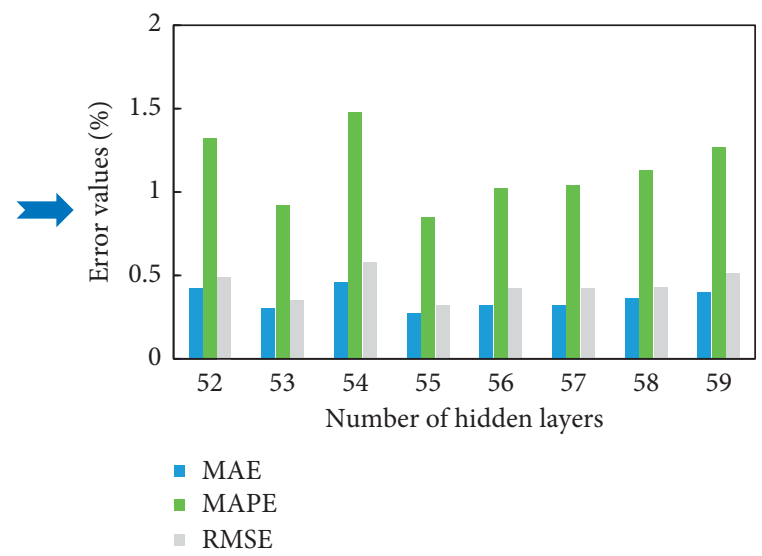

(d)

Figure 2: The optimization results of the hidden layer in the ELM model. (a) Stiffness modulus/20-70 layers. (b) Stiffness modulus/60-68 layers. (c) $m$-value/20-70 layers. (d) $m$-value/52-59 layers.

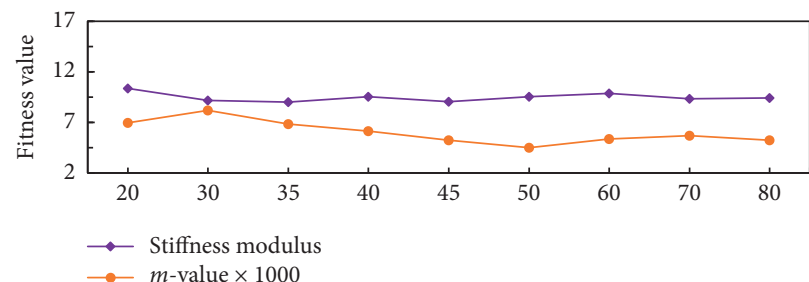

(a)

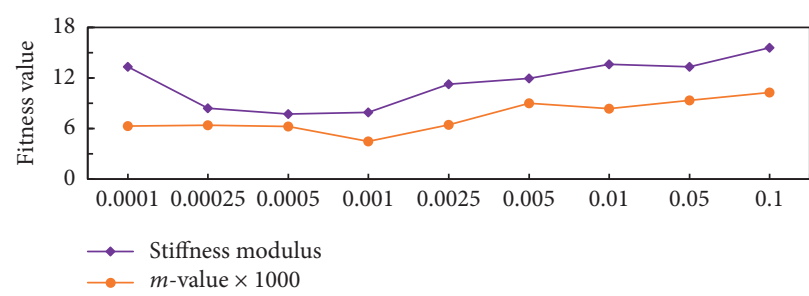

(c)

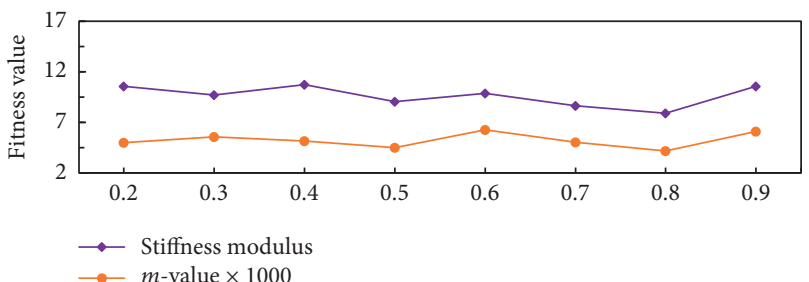

(b)

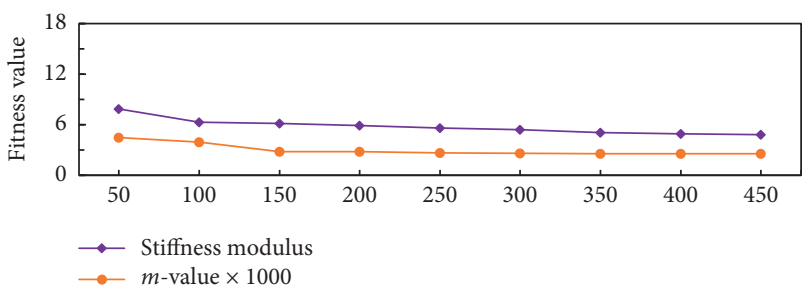

(d)

Figure 3: The parameter optimization results of the GA. (a) Population size. (b) Crossover probability. (c) Mutation probability. (d) Maximum iterations. 

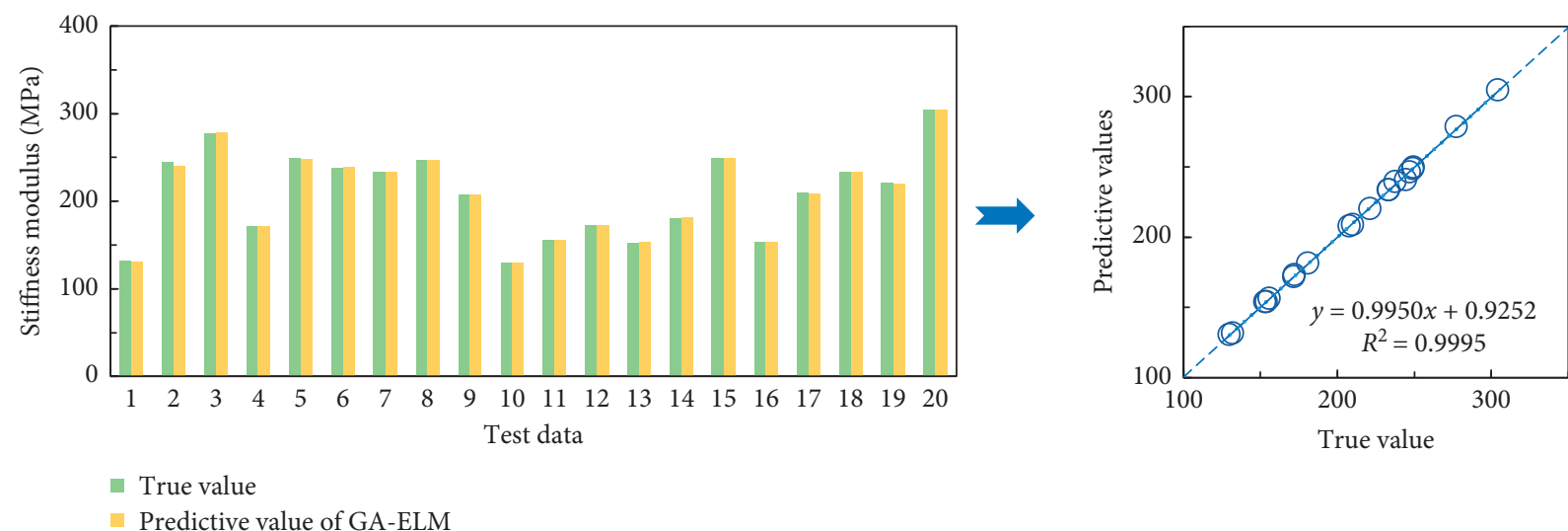

- Predictive value of GA-ELM

(a)
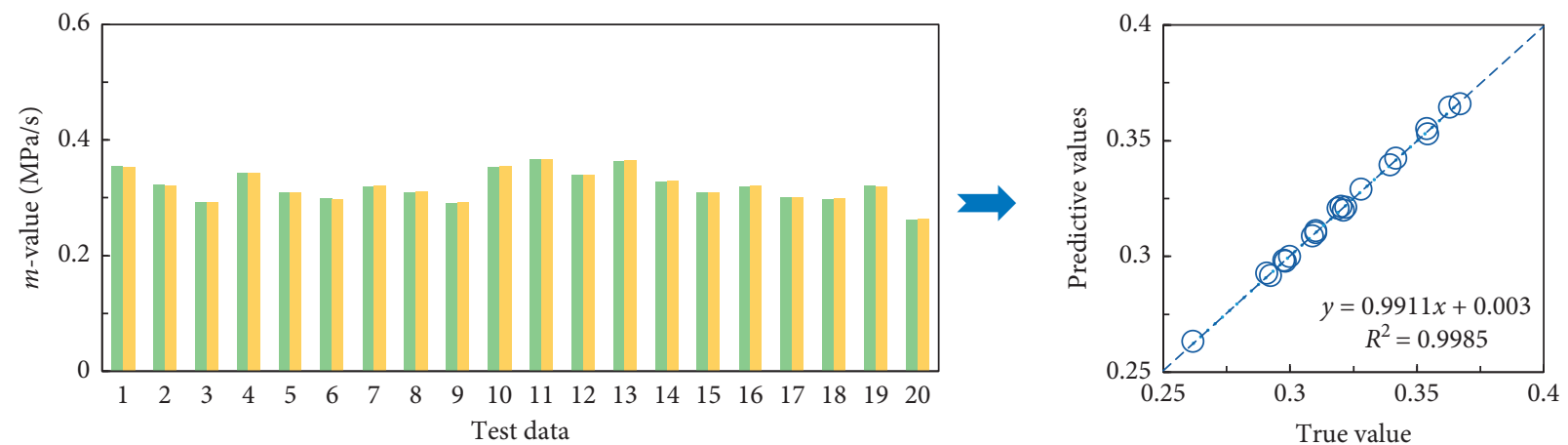

- True value

1- Predictive value of GA-ELM

(b)

Figure 4: Accuracy of the GA-ELM prediction model. (a) Stiffness modulus. (b) $m$-value.

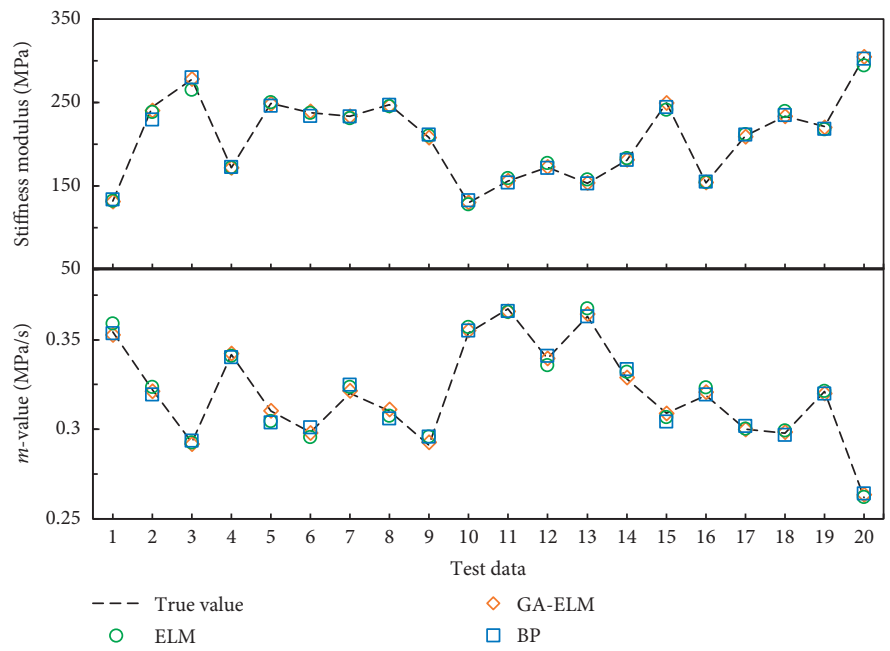

(a)
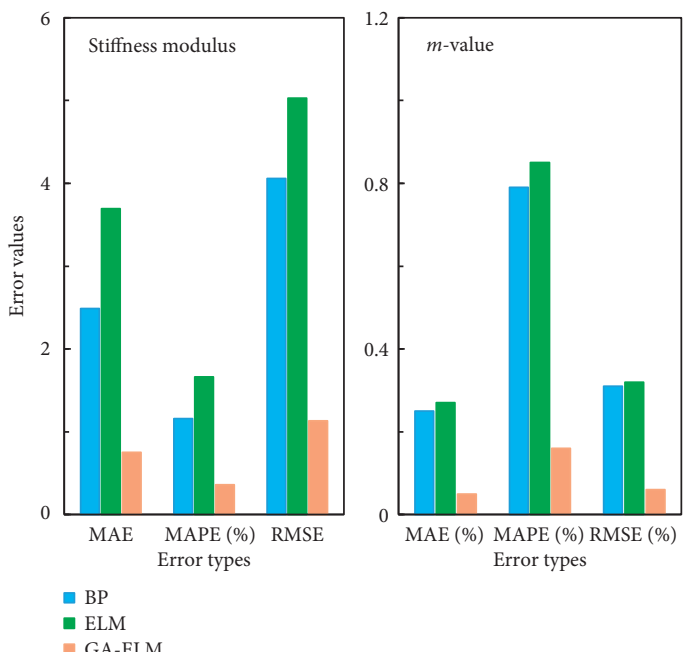

(b)

Figure 5: Prediction accuracy comparison of different models. (a) Predictive value comparison. (b) Error comparison. 
$0.7539,0.36 \%$, and 1.1323 , respectively. Compared with the BP model and the ELM model, the error of the GA-ELM model decreased by $68.97-72.10 \%$ and $83.63-87.41 \%$, respectively. And for the $m$-value, the MAE, MAPE, and RMSE of the GA-ELM model were 0.0005, $0.16 \%$, and 0.0006, respectively. Compared with the BP model and the ELM model, the error of the GA-ELM model decreased by $79.75-80.65 \%$ and $81.18-81.48 \%$, respectively. This indicated that the GA-ELM model had better advantages in prediction accuracy and efficiency than the traditional prediction model.

\section{Conclusion}

(1) The GA-ELM prediction model can rapidly predict the low-temperature rheological properties of SBS modified asphalt. Compared with the traditional prediction model, the GA-ELM model had obvious accuracy and efficiency. The error was reduced by $68.97-81.48 \%$.

(2) The applicable boundaries of the input parameter are as follows. For the matrix asphalt, PG-grade is 58-22 and 58-16; stiffness modulus is $265-320 \mathrm{MPa}$; $m$ value is $0.19-0.3 \mathrm{MPa} / \mathrm{s}$; asphaltenes are 7.24-15.52\%; colloids are 19.35-33.21\%; aromatics are $35.55-46.70 \%$; and saturates are $19.19-25.24 \%$. For the SBS modifier, block ratio (S/B) is $40 / 60$ and $30 / 70$; tensile strength is over $12 \%$; elongation is over $590 \%$; permanent deformation is less than $45 \%$; hardness is over 60; and the content is 3.6-5.2\%.

(3) Due to the use of the genetic algorithm, the training efficiency of the GA-ELM model was lower than that of the original ELM model. In addition, the optimum number of the hidden layers was influenced by the input of data. The more the number and type of data, the less the optimum number of hidden layers. However, the optimum number will remain within a specific range.

(4) The GA-ELM model still belongs to the black-box operation. It should be further improved in future studies. With the updating of the algorithm and software, the prediction model will be established based on input parameters, which is more conducive to regulating the model and the feedback of the results. In addition, more raw material parameters and their corresponding performance indexes should be collected in the future to further expand the prediction range of the model.

\section{Data Availability}

The data used to support the findings of this study are included within the article.

\section{Conflicts of Interest}

The authors declare that they have no conflicts of interest regarding the publication of this paper.

\section{Acknowledgments}

This research was sponsored by the Natural Science Foundation of Xinjiang Uygur Autonomous Region (2020D01A92), Fundamental Research Funds for the Central Universities, CHD (300102219701 and 300102219314), Science and Technology Project of Transportation Industry in Xinjiang Uygur Autonomous Region (2019-ZD1-016), and China Postdoctoral Science Foundation (2020M683709XB).

\section{References}

[1] S. Rezaei, H. Ziari, and S. Nowbakht, "Low temperature functional analysis of bitumen modified with composite of nano- $\mathrm{SiO}_{2}$ and styrene butadiene styrene polymer," Petroleum Science and Technology, vol. 34, no. 5, pp. 415-421, 2016.

[2] R. Tarefder and S. Yousefi, "Rheological examination of aging in polymer-modified asphalt," Journal of Materials in Civil Engineering, vol. 28, no. 2, Article ID 04015112, 2016.

[3] L. Shan, X. Qi, X. Duan, S. Liu, and J. Chen, "Effect of styrenebutadiene-styrene (SBS) on the rheological behavior of asphalt binders," Construction and Building Materials, vol. 231, Article ID 117076, 2020.

[4] Z. Chen, H. Zhang, H. Duan, and C. Wu, "Determination of time-temperature superposition relationship of SBS modified asphalt based on special rheological phenomenon caused by SBS-formed structure in asphalt matrix," Construction and Building Materials, vol. 260, Article ID 119835, 2020.

[5] S. Ren, X. Liu, M. Li, W. Fan, J. Xu, and S. Erkens, "Experimental characterization of viscoelastic behaviors, microstructure and thermal stability of CR/SBS modified asphalt with TOR," Construction and Building Materials, vol. 261, Article ID 120524, 2020.

[6] B. V. Kök, M. Yilmaz, and A. Geçkil, "Evaluation of lowtemperature and elastic properties of crumb rubber- and SBSmodified bitumen and mixtures," Journal of Materials in Civil Engineering, vol. 25, no. 2, pp. 257-265, 2013.

[7] W. Huang, X. Fu, Y. Li, and S. Liu, "Evaluation of low temperature performance and correlation analysis on low temperature indexes of SBS modified asphalts," Journal of Building Materials, vol. 20, no. 3, pp. 456-463, 2017, in Chinese.

[8] X. Ma, H. Chen, X. Zhang, M. Xing, P. Yang, and Z. Wang, "An analysis over the correlation between low temperature rheological properties of SBS-modified asphalt and raw materials' performance parameters," Materials Review, vol. 32, no. 22, pp. 3885-3890, 2018, in Chinese.

[9] Q. Chen, C. Wang, P. Wen, M. Wang, and J. Zhao, "Comprehensive performance evaluation of low-carbon modified asphalt based on efficacy coefficient method," Journal of Cleaner Production, vol. 203, pp. 633-644, 2018.

[10] Q. Chen, C. Wang, Z. Qiao, and T. Guo, "Graphene/tourmaline composites as a filler of hot mix asphalt mixture: preparation and properties," Construction and Building Materials, vol. 239, Article ID 117859, 2020.

[11] H. Liu, A. Sha, Z. Tong, and J. Gao, "Autonomous microscopic bunch inspection using region-based deep learning for evaluating graphite powder dispersion," Construction and Building Materials, vol. 173, pp. 525-539, 2018.

[12] Y. Cao, A. Sha, Z. Liu, J. Li, and W. Jiang, "Energy output of piezoelectric transducers and pavements under simulated 
traffic load," Journal of Cleaner Production, vol. 279, Article ID 123508, 2021.

[13] Q. Chen, S. Wang, C. Wang et al., "Modified waterborne epoxy as a cold pavement binder: preparation and long-term working properties," Journal of Materials in Civil Engineering, vol. 33, 2021.

[14] C. Yan, W. Huang, P. Lin, Y. Zhang, and Q. Lv, "Chemical and rheological evaluation of aging properties of high content SBS polymer modified asphalt," Fuel, vol. 252, pp. 417-426, 2019.

[15] M. Xu, Y. Zhang, P. Zhao, and C. Liu, "Study on aging behavior and prediction of SBS modified asphalt with various contents based on PCA and PLS analysis," Construction and Building Materials, vol. 265, Article ID 120732, 2020.

[16] A. Diab, Z. You, X. Li, J. C. Pais, X. Yang, and S. Chen, "Rheological models for non-Newtonian viscosity of modified asphalt binders and mastics," Egyptian Journal of Petroleum, vol. 29, no. 2, pp. 105-112, 2020.

[17] Q. Chen, C. Wang, X. Sun, Y. Cao, T. Guo, and J. Chen, "Evaluation and prediction for effect of conductive gussasphalt mixture on corrosion of steel bridge deck," Construction and Building Materials, vol. 228, Article ID 116837, 2019.

[18] R. Prabu and R. Harikumar, "A performance analysis of GAELM classifier in classification of abnormality detection in electrical impednce tomography (EIT) lung images," Journal of Scientific and Industrial Research, vol. 75, pp. 404-411, 2016.

[19] NB/SH/T 0509-2010, Test Method for Separation of Asphalt into Four Fractions, China Petrochemical Press, Beijing, China, 2010.

[20] Y. Mei, Q. Sun, L. Yu, C. Wang, and H. Xiao, “Grain size prediction of aluminum alloy dies castings based on GAELM," Acta Metallurgica Sinica, vol. 53, no. 9, pp. 1125-1132, 2017, in Chinese. 